\title{
Le musée d'Ethnographie de Neuchâtel :une muséographie impertinente au service des publics
}

\author{
Serge Chaumier, Professeur à l'université d'Artois, Samuel Cordier, \\ Directeur de l'Ocim, Marc-Olivier Gonseth, Directeur du musée \\ d'Ethnographie de Neuchâtel, Société, inclusif et expérience
}

\section{OpenEdition Journals}

Édition électronique

URL : http://journals.openedition.org/ocim/2685

DOI : $10.4000 /$ ocim.2685

ISSN : 2108-646X

Éditeur

OCIM

Édition imprimée

Date de publication : 1 juillet 2018

Pagination : $32-41$

ISSN : 0994-1908

Référence électronique

Serge Chaumier, Professeur à l'université d'Artois, Samuel Cordier, Directeur de l'Ocim, Marc-Olivier Gonseth, Directeur du musée d'Ethnographie de Neuchâtel, Société, inclusif et expérience, « Le musée d'Ethnographie de Neuchâtel :une muséographie impertinente au service des publics », La Lettre de I'OCIM [En ligne], 178 | 2018, mis en ligne le 01 juillet 2019, consulté le 06 septembre 2019. URL : http://journals.openedition.org/ocim/2685; DOI : 10.4000/ocim.2685

Ce document a été généré automatiquement le 6 septembre 2019.

Tous droits réservés 


\title{
Le musée d'Ethnographie de Neuchâtel :une muséographie impertinente au service des publics
}

\author{
Serge Chaumier, Professeur à l'université d'Artois, Samuel Cordier, \\ Directeur de l'Ocim, Marc-Olivier Gonseth, Directeur du musée \\ d'Ethnographie de Neuchâtel, Société, inclusif et expérience
}

1 Pour commencer, pouvez-vous retracer le parcours qui vous a conduit à la direction du MEN ?

2 Je suis né dans le Jura, un canton limitrophe de celui de Neuchâtel. J'avais l'intention de me diriger vers l'ethnologie, l'archéologie et la linguistique et il se trouve que ces disciplines étaient enseignées à l'université de Neuchâtel, raison pour laquelle j'ai débarqué ici en 1972. J'arrive alors que le Musée et l'enseignement de l'ethnologie sont encore dans les mains de Jean Gabus (1908-1992). À l'université, je suis obligé de passer par la case géographie car il n'y a pas encore de formation complète en ethnologie, mais seulement trois semestres de spécialisation. Pierre Centlivres prend la direction de l'Institut d'ethnologie en 1974 et développe une tout autre manière d'envisager la discipline. Il amène notamment les courants théoriques du moment, qui n'intéressaient pas spécialement Jean Gabus, dont l'enseignement était resté centré sur la culture matérielle.

En fait, l'arrivée de Pierre Centlivres à Neuchâtel correspond à un changement de paradigme. Il prend Jacques Hainard comme chef de travaux et lorsque Jean Gabus quitte la direction du musée en 1978, en laissant derrière lui une équipe qui ne sait pas trop comment gérer l'exposition annuelle et envisage de s'en remettre à ses collègues bâlois, les deux hommes vont voir les autorités politiques responsables pour leur signifier qu'ils sont parfaitement capables d'enseigner à l'université et de prendre parallèlement en charge l'exposition annuelle du musée. Donc au lieu d'avoir un responsable de musée qui enseigne l'ethnologie à l'université, situation antérieure, nous avons un professeur d'ethnologie et son bras droit qui prennent en charge l'exposition du musée. J'entends parler de ce projet à la fin d'un cours donné par Pierre Centlivres, qui propose aux étudiants intéressés, parmi la soixantaine qui suivent son enseignement, de réfléchir avec 
lui sur le thème du nomadisme. L'idée de m'investir dans un projet d'exposition à la fin de mes courtes études d'ethnologie et au retour d'un voyage de six mois en Irlande me plaisait bien et, surtout, j'avais repéré en Pierre Centlivres un enseignant passionnant et en Jacques Hainard un personnage dynamique et attachant.

Donc, en 1979, je réalise mon premier contrat d'ethnomuséographie dans le cadre de l'exposition Être nomade aujourd'hui. Je produis d'abord une analyse puis un texte articulé sur les beatniks, hippies et routards, que l'équipe accepte d'intégrer au catalogue. Ensuite, ils me proposent de prendre en charge une section sur ce thème dans l'exposition ellemême. Je me lance alors avec d'autres dans la réalisation de la galerie de $200 \mathrm{~m} 2$ qui termine le parcours. L'équipe du musée est très prise par le nomadisme classique et me laisse donc développer très librement mon sujet avec une assistante scénographe, sur les thèmes de la musique, du vêtement et des mouvements de contestation. J'arrive à donner une cohérence à mon propos et en même temps, je fais de "la route" mon mémoire de licence. C'est comme ça que j'ai été lâché de manière très libre dans un projet à la fois créatif et formateur.

Et comment poursuivez-vous?

6 Le virus de l'exposition m'a pris dès cette première expérience. Comme je m'étais fait connaître à cette occasion, j'ai d'abord été engagé pour mener une enquête en sociologie rurale dans une région de montagne, puis pour participer à un projet de recherches sur l'alimentation. En 1983, à la demande de Jacques Hainard, qui a pris la direction du musée et qui cherche de l'aide pour développer un projet d'exposition théorique sur les objets, Pierre Centlivres me propose un demi-poste d'assistant qu'il met en lien avec l'exposition en question. L'Institut me délègue donc à temps plein sur le projet intitulé Objets prétextes, objets manipulés. Je recommence alors mes études d'ethnologie - car à chaque fois que je me lance dans une expo, je recommence mes études - afin d'acquérir les connaissances que je n'ai pas dans un champ que je défriche. Et cela donne un texte synthétique et une exposition qui alimentera longtemps notre démarche, puisqu'elle est fondatrice de ce qui deviendra notre ligne. Nous sommes en 1984, Jacques Hainard est tout frais, il n'a pas encore théorisé la rupture, mais l'idée est en gestation et arrivera à maturité trois ans plus tard. J'arrive donc dans un milieu intellectuel en train de se transformer, de chercher une forme, une ligne et un cadre théorique. Et comme j'aime bien lire, écrire, synthétiser, imaginer et ensuite spatialiser, je n'ai plus vraiment d'autres envies que celle-là. Je continue quand même de faire du terrain, notamment aux Philippines, car Pierre Centlivres me pousse à terminer ma thèse pour pouvoir postuler à sa succession. Mais je lui annoncerai bientôt que j'ai choisi le musée.

Vous avez hésité entre les deux?

8 Pendant une période, j'ai ressenti un balancement entre l'Institut et le musée, entre une démarche théorique et l'organisation d'un discours dans l'espace. Avec Jacques Hainard, nous nous étions très bien entendus sur Objets prétextes, mais une fois l'exposition inaugurée, j'ai ressenti comme une dépossession : mon travail était passé sous silence, je n'étais plus invité à rien, je ne faisais plus partie de l'équipe. Pendant deux ou trois années, je me suis donc à nouveau centré sur l'ethnologie. En 1986, Jacques Hainard m’a cependant proposé un nouveau mandat pour l'exposition Des animaux et des hommes (1987). Après cette troisième expérience, je deviens collaborateur scientifique régulier des expositions du MEN. Je me forme donc au sein d'une école en train se créer et je contribue à cette mise en place, mais je n'ai pas de réelle assise. J'obtiens cette assise en 1992, lorsque Jacques Hainard me propose un mi-temps après le départ d'une collaboratrice. Je 
fais dès lors partie du groupe à part entière, je pèse plus fortement sur les projets et je peux enfin suivre la totalité des activités muséales. Car si la création d'une exposition est un processus formateur, faire vivre une exposition propose une tout autre expérience. direction du musée. J'avais travaillé avec eux avant le départ de Jacques. Yann est arrivé
lors de la préparation de La Grande Illusion (2000-2001) et Grégoire a commencé à collaborer à la fin de $X$ : spéculations sur l'imaginaire et l'interdit (2003). Tous deux se sont par ailleurs beaucoup investis dans la préparation des manifestations liées au Centenaire du musée (2004), un épisode-clé dans la formation de l'équipe du MEN. Donc j'avais en eux des personnes à la fois compétentes sur le plan de l'expographie et capables d'apporter un plus dans le domaine des activités parallèles, l'un étant plus orienté son et musique et l'autre davantage image fixe et animée. Mais pour répondre à la question, il y a en effet filiation dans le fait d'entrer dans une structure à travers un projet, d'y développer des compétences, de s'y faire reconnaître. Dès que j'ai été nommé à la direction, j'ai donc proposé à Yann et Grégoire de rejoindre mon équipe à $50 \%$ chacun puisque je devais repourvoir mon propre poste. Nous nous sommes alors lancés ensemble dans l'exposition Figures de l'artifice (2006-2008). et recherches.

Et vous arrivez à maintenir la production d'expositions, avec une partie cours, formation

On doit cette spécificité du tandem musée-université à Jean Gabus. Développant une ligne muséographique puissante dopée par son aura diplomatique, il a parallèlement réussi à relancer un enseignement en ethnographie après plus de 30 ans d'abandon. Le fait qu'il ait recréé cette poche d'enseignement a permis à Pierre Centlivres de faire basculer l'ethnographie muséale enseignée jusque là du côté de l'ethnologie. Et Jacques Hainard a fait de même au musée en développant une position de rupture. J'ai continué sur cette lancée dans la mesure où j'ai accordé une importance toute particulière à notre lien à l'Institut d'Ethnologie, développant notamment une longue collaboration autour de la question du patrimoine culturel immatériel, sans rien lâcher sur le plan des exigences expographiques. Pour aller vite, on pourrait dire que nos contributions successives sont parvenues à créer une véritable école de muséographie, originale, critique et alimentée par le fait social et muséal. 


\begin{tabular}{|c|c|c|c|c|c|c|c|c|}
\hline t & a & 5 & 5 & c & 桖 & $6 \mathrm{~m}$ & 0 & 4NIII \\
\hline 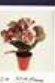 & $\$$ & $\Delta$ & 5 & $I$ & \% & $\dot{8}$ & t & 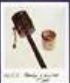 \\
\hline , & 6 & 6 & 7 & \& & 1 & 8 & 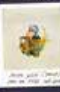 & 8 \\
\hline 8 & $x$ & 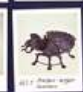 & 8 & 0 & 10 & 新 & T & 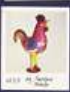 \\
\hline 国 & 60 & 8 & 8 & 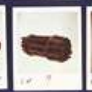 & 1 & 越 & 1 & 2 \\
\hline $\boldsymbol{a}$ & 1 & 吅 & $\frac{3}{8}$ & a & 8 & 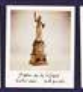 & D & $k$ \\
\hline 数 & 1 & 2 & V & $\theta$ & 0 & 1 & $\theta$ & $\tilde{2}$ \\
\hline 28 & $\underline{E}$ & 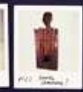 & If & A & 6 & $\approx$ & 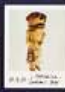 & $\mathcal{P}$ \\
\hline & 8 & 16 & 1 & 4 & 8 & 2 & 8 & \\
\hline
\end{tabular}

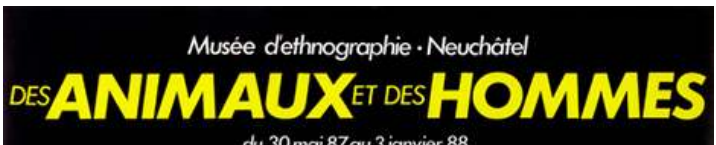

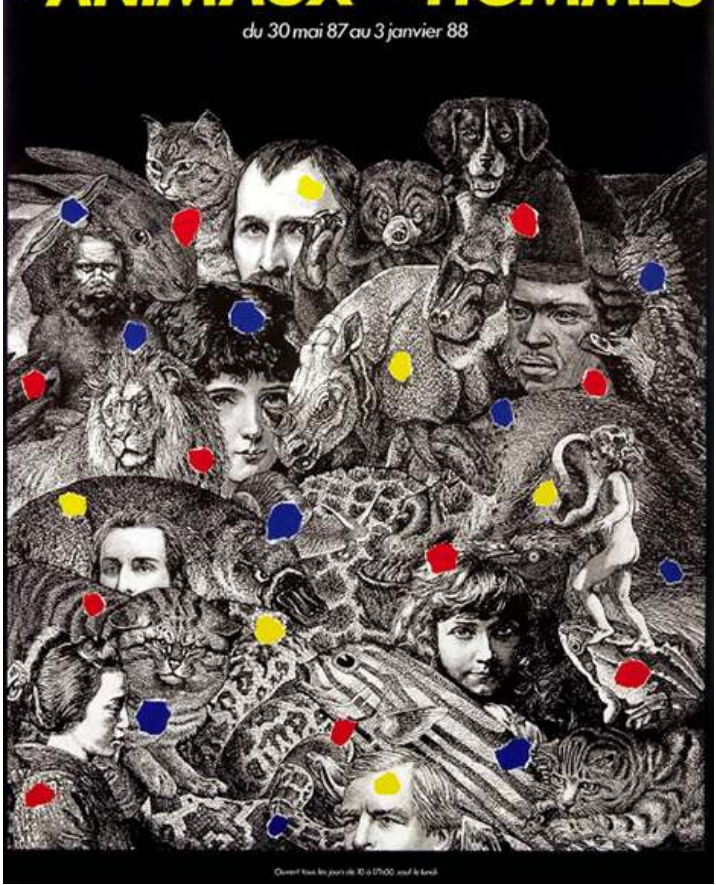



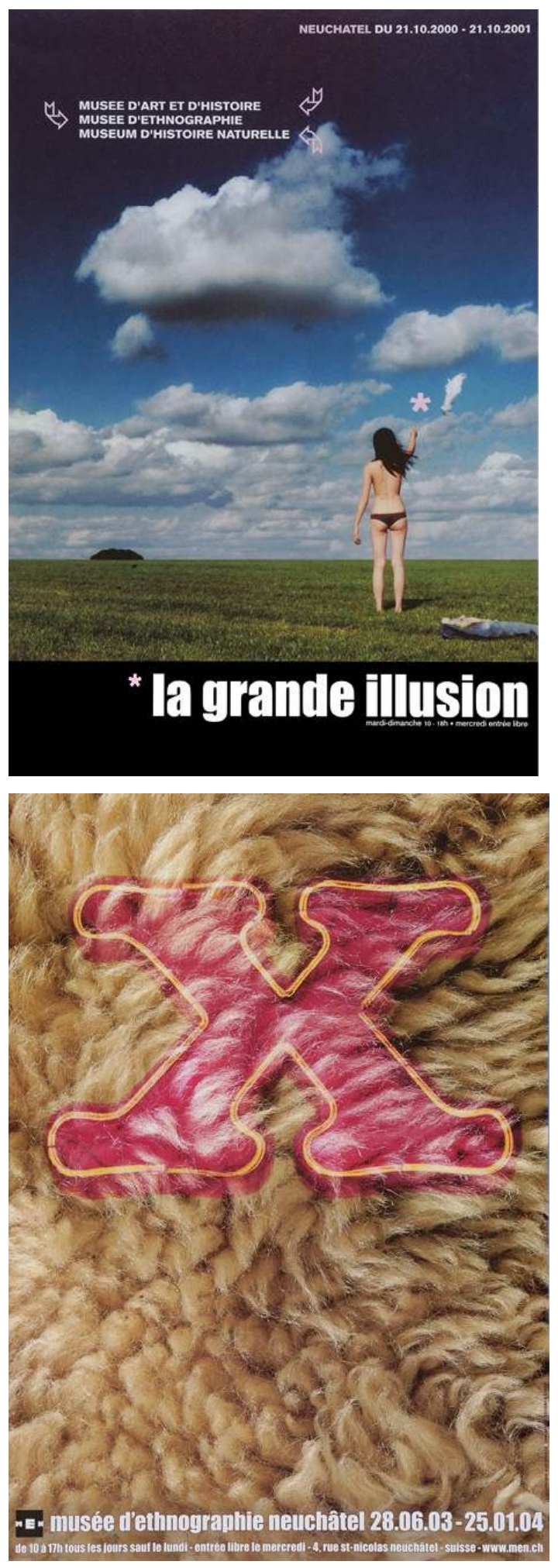

15 Pour revenir sur le passage, l'exposition de transition entre Jacques et le moment où vous prennez la direction, c'est Remise en boîtes. Ce passage est symboliquement marqué et marquant. Pour vous la dernière exposition temporaire que vous faites est Secrets, mais elle se fait en extérieur, dans la ville.

16 Remise en boîtes ponctue magnifiquement le cycle développé avec Jacques Hainard. Cette exposition empoigne un sujet que nous avions repoussé jusque là, qui est notre rapport à 
la mémoire et à l'histoire : le lien au témoin, au passé, à la résurgence, au traumatisme, avec en ligne de mire les travaux de Robert Hertz sur les doubles funérailles, et finalement le rapport à la mort. Cette exposition propose donc une réflexion générale sur la question du souvenir et de la fin des choses. Quant à Secrets (2015), un élément de réponse est lié à l'histoire des projets. Les trois expositions Bruits (2010), Hors-champ (2012), et Secrets ont été prévues cinq ans plus tôt dans un canevas de recherche avec l'Institut d'ethnologie sur le Patrimoine culturel immatériel proposé au Fonds national de la recherche scientifique. Un autre élément est qu'on pensait que le bâtiment des expositions temporaires (ou Black-box) serait en travaux, donc qu'il ne serait pas disponible, et qu'il faudrait donc aller jouer à l'extérieur. Le fait de terminer mon cycle d'expositions temporaires par une proposition développée au plus près de la population neuchâteloise m'a beaucoup plu : je cassais enfin le côté "tour d'ivoire" de la colline et j'engageais un rapport différent avec les autorités, les structures, les collègues, car on n'a pas pour habitude de prendre toute une ville comme terrain d'exercice, et ça c'était super fort.

Depuis 2006, est ce qu'il y a des moments particuliers à retenir, qui vous ont marqué ? Dans les expositions est ce qu'il y en a une qui se détacherait comme un bon ou un moins bon souvenir, mais qui aurait permis de passer un cap?

La vraie transition, c'est l'exposition Figures de l'artifice en 2006 ! Moi qui ai toujours été dans l'ombre de Jacques Hainard, je devais reprendre une ligne qui m'appartenait autant qu'à lui, sans en être le porteur légitime. Et bien entendu on m'attendait au contour. Je devais réagir en peu de temps et également reconstituer une équipe, car il ne faut pas oublier que si Jacques Hainard était parti, mes collègues Roland Kaehr et François Borrel étaient eux aussi sur le départ. Et je devais prouver que j'étais capable de tenir un projet, un chantier d'exposition, un budget et une équipe nouvelle à la fois à l'intérieur (conception) et à l'extérieur (réalisation).

C'est après avoir réalisé Retour d'Angola (2007) que nous avons lancé le chantier des collections qui aboutira dix ans plus tard à la résurrection complète de la Villa. Donc s'il y a un pas décisif vers une rénovation non seulement des bâtiments, mais aussi de nos manières de faire, c'est bien de Retour d'Angola qu'il s'agit.

21 Le deuxième moment-clé, c'est le bouclement de mon premier cycle quadriennal car c'est à ce moment que s'est jouée ma crédibilité au niveau organisationnel et budgétaire. Sinon, je dirais que toutes les expositions faites depuis que je travaille au MEN représentent à leur manière des moments-clés car elles ont toujours cristallisé des avancées, proposé des paliers et surtout permis aux équipes de gestion, de conception et de réalisation de se retrouver.

La renovation de la Villa constitue le troisième moment fort que j'extrairais du lot même si elle correspond à un des moments les plus difficiles que j'ai vécus dans ma carrière. L'équipe s'est en effet retrouvée éclatée sur plusieurs lieux et j'ai peu à peu senti la distance s'installer puis le fossé se creuser entre les personnes. Il a fallu la réouverture de la Villa et la mise en place de l'exposition de référence pour que nous retrouvions l'effet d'une équipe travaillant en osmose.

23 Ce qui caractérise les expositions de Neuchâtel, c'est une liberté de ton, que l'on ne voit nul par ailleurs, qui semble impossible en France. On se souvient de Helvetia Park. Vous allez très loin, comment pouvez- vous avoir une telle liberté de ton? 
Un émissaire de la Confédération est quand même venu au MEN avant l'inauguration d' Helvetia Park parce qu'ils avaient entendu parler d'un jeu de massacre et qu'ils voulaient s'assurer que nous ne mettions pas le Président de la Confédération en mauvaise posture ! Le contrôle existe donc quand même, mais il est vrai que nous jouons avec cette dimension. À Neuchâtel, nous avons la chance d'avoir depuis longtemps la confiance des autorités communales et plus précisément de la Direction de la culture, qui est notre seule instance de surveillance et de tutelle. Neuchâtel est une petite ville plutôt de centre gauche, ce qui n'est pas commun en Suisse, et qui s'est fait un point d'honneur à laisser une liberté de penser, et même de critiquer, à ses acteurs culturels. Il se trouve que le MEN a pris sur ce plan un rôle majeur et comme il y a avait de fortes têtes à sa direction, l'idée s'est imposée que le mode de réflexion qu'elles proposaient n'était pas à réprouver, mais représentait au contraire un vrai potentiel de dynamisation, à l'interne comme à l'externe. L'intérêt pris à l'extérieur pour ce petit musée de province provient donc beaucoup de sa liberté de ton. Il ne vient en effet pas de la qualité exceptionnelle de ses collections, ni de moyens mirobolants mis en place au niveau des technologies interactives! Non, il vient de son insolence, de sa capacité à ne pas se prendre trop au sérieux et de sa volonté de prendre au sérieux ceux qui le visitent. Il s'agit donc essentiellement d'un jeu que nous essayons d'instaurer pour affuter le regard et développer une sorte de curiosité critique.

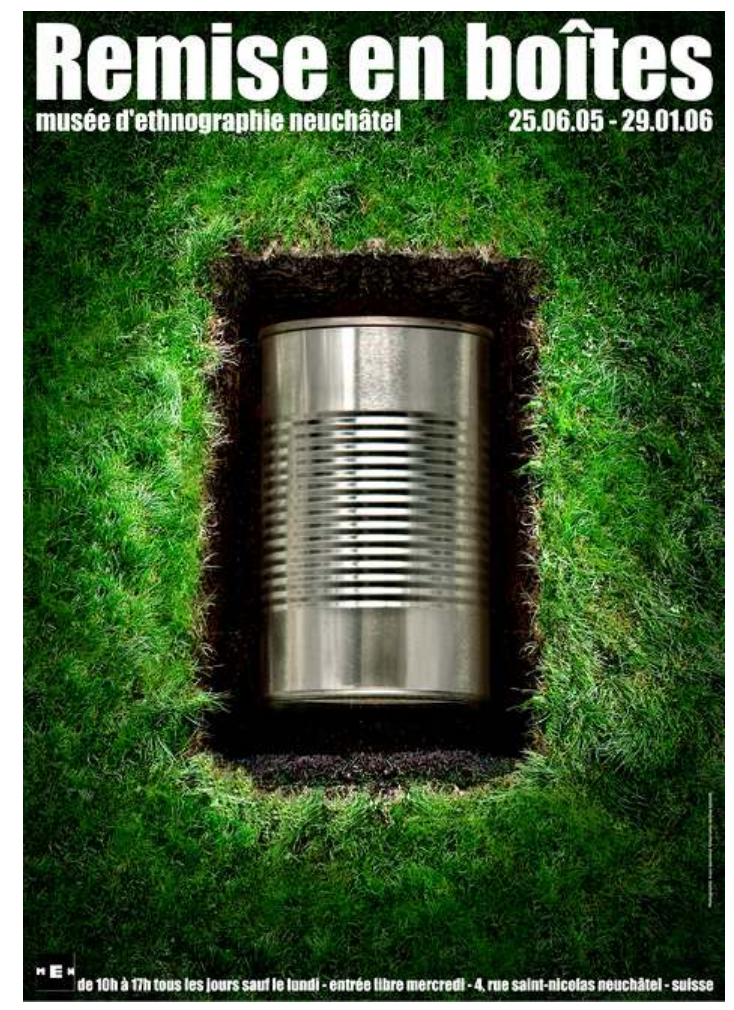



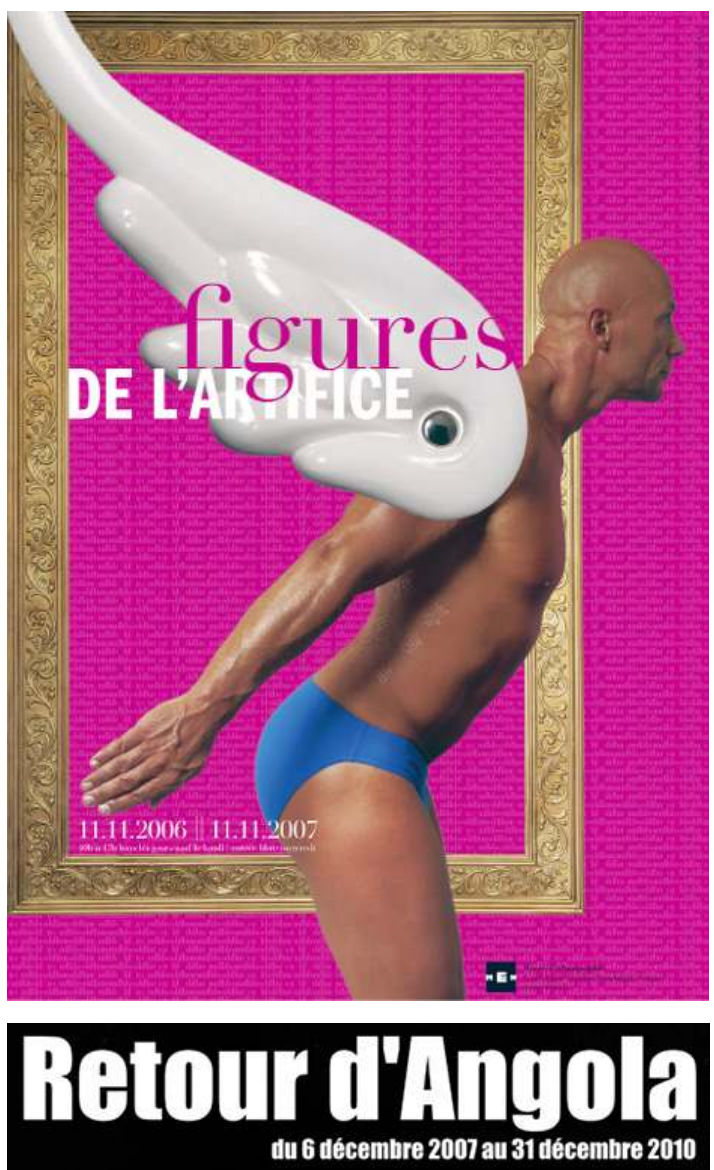

du 6 décembre 2007 au 31 décembre 2010

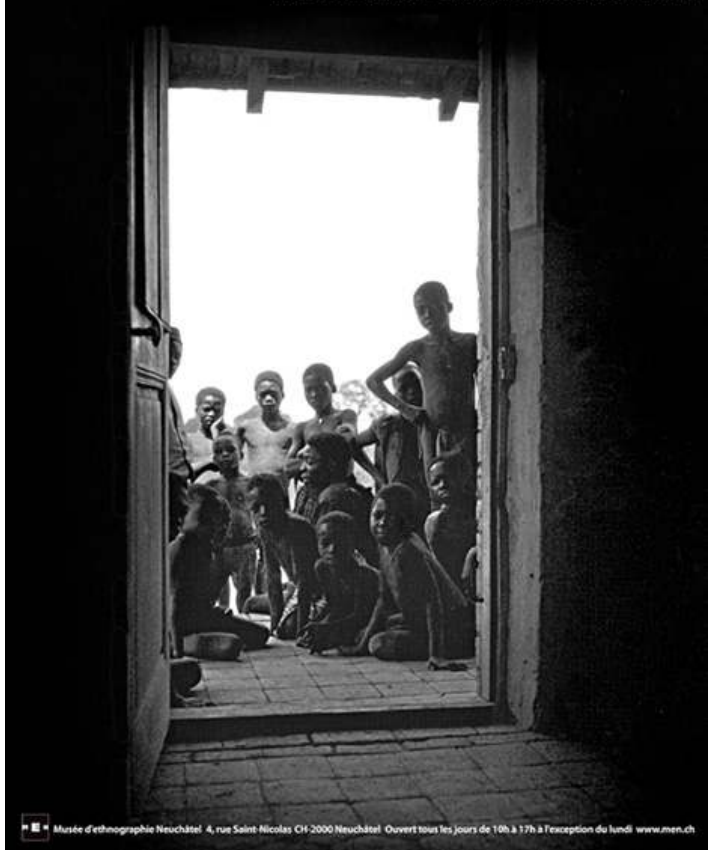




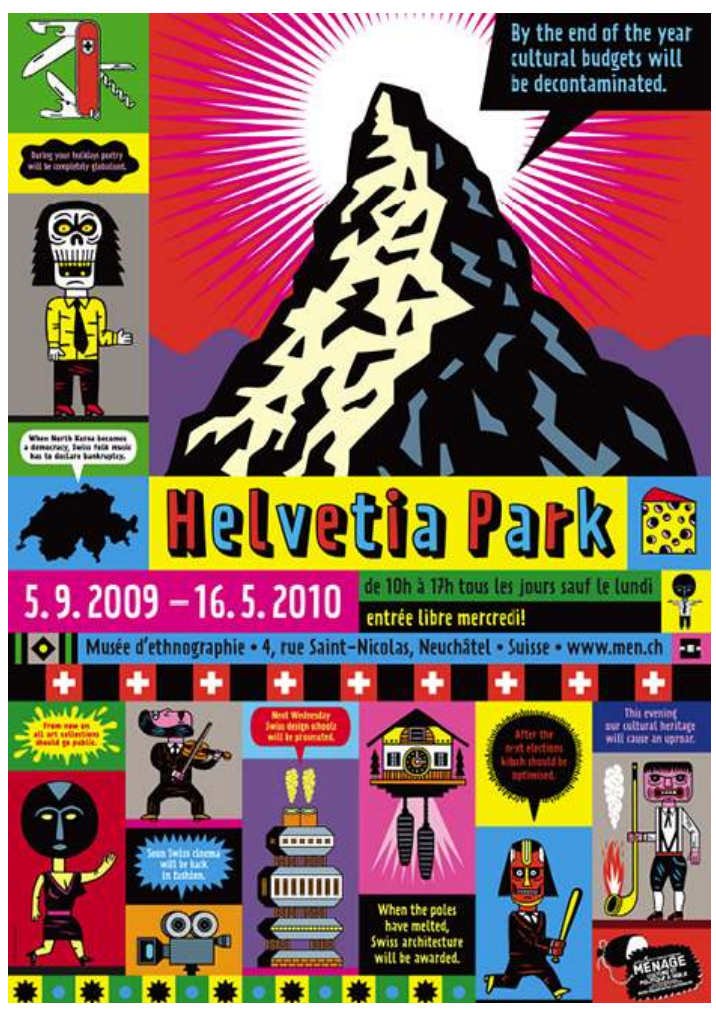

Existe-t-il des spécificités à Neuchâtel liées à la gestion des équipes ? Sur le management ? Dans le choix de confier des projets à certains, ou le projet de faire travailler des personnes, comment arrivez-vous à favoriser une forme de créativité ?

Concernant la gestion de l'équipe, ma manière consiste à impliquer au maximum tous les membres de l'équipe à travers des projets qui leur correspondent, en laissant à chaque personne une grande autonomie. Mais si nous fonctionnons de manière très démocratique dans les périodes normales, les exigences liées aux expositions donnent aussi généralement lieu à une période de dictature éclairée.

Vous êtes combien dans l'équipe?

Il y a actuellement huit personnes représentant 5 ETP dans l'équipe scientifique. Pendant plusieurs années, j'ai eu tendance à valider l'ancien schéma hérité de Jacques Hainard, avec un petit groupe s'occupant des expositions et un autre des collections, même si tout le monde était associé au montage final des expositions. Ce n'était pas une bonne solution, je le savais, mais elle a permis à l'ancienne équipe de bien fonctionner. Dès que j'ai pu faire sauter ce verrou, je l'ai fait. D'abord en impliquant le groupe des collections dans de petites expositions, sous forme de carte blanche. Puis en les impliquant plus tôt dans la réflexion. Mais c'est l'exercice de la rénovation de la Villa qui m'a permis de concerner chacun, d'impliquer la totalité de l'équipe dès le départ et de partager les responsabilités collectivement. J'ai souhaité d'abord intéresser très tôt l'équipe au projet, ensuite, par petits groupes, donner des responsabilités partagées, et enfin initier un échange rapide et constant avec les scénographes, arbitré par moi.

L'intégration du scénographe dès le début du projet est fondamentale pour vous?

Ce que l'on appelle ici le ping-pong avec les scénographes est un élément majeur de notre fonctionnement et représente notre vraie différence par rapport à bien des équipes. Sur la totalité du processus, il existe peut être 5 ou 6 couches de constructions différenciées de l'exposition, mais toutes sont discutées, modifiées et renvoyées au fur et à mesure de 
nos échanges. Les points de vue sont démultipliés par huit personnes et, évidemment, toutes ne peuvent être en relation directe avec les scénographes, sinon c'est le cauchemar. Cette relation est donc sous ma responsabilité. Réciproquement, il est également de ma responsabilité que tout ce qui émane des scénographes soit rediscuté dans le groupe de conception.

Faire une exposition "permanente", entre guillemets parce qu'elle est évolutive, à partir de pièces contemporaines, c'est gonflé. N'importe quel musée d'ethnographie qui se rénove le fait à partir de ses trésors, ses chefs d'œuvre, ses pièces maitresses, ses "Jocondes".

Pour moi, ce qui serait gonflé au sens de "casse-gueule ", ce serait de nous centrer sur nos chefs-d'œuvre! Car partir de là reviendrait à nous renier complètement, à nager à contre courant de tout ce que nous avons entrepris dans le domaine de l'exposition depuis que je suis dans la profession. En revanche, nous avons associé différents types d'objets dans les différents espaces afin de créer une médiation, un appel d'air entre le passé et le présent. Cette accroche avec le contemporain permet d'aller plus loin dans l'histoire et de concerner davantage nos visiteurs, j'en suis certain. À mon sens, consommer les objets à travers la seule historiographie classique est ennuyeux.

Mais justement, dans ces trois phases que vous décrivez ("texte", "image", "objet"), vous dites que c'est inversé, cela voudrait dire que dans la villa, vous êtes quand même partis des objets. Or, a priori, vous ne partez pas de collections, mais d'une idée de découpage.

Vous me piégez dans ma propre théorie! Mais quand nous choisissons de partir d'une mosaïque, d'impliquer le contemporain et de travailler de manière modulaire, c'est bien d'idées qu'il s'agit. Nous avons décidé d'un mode, d'un cadre, d'une dynamique de lecture de nos collections : c'est donc bien ce que j'appelle "théoriser", même si la piste de départ consiste à retourner aux collections et non à développer une thématique. Partant de cette règle, nous nous sommes demandé ensuite ce qui pourrait bien unifier notre propos. Nous avons évoqué l'idée de voyage, qui aurait pu être une thématique unificatrice. Mais finalement nous avons décidé de renoncer à notre pratique habituelle et d'oser la mosaïque. Et nous en sommes arrivés à des termes évocateurs tels que "Au-delà", "Poids", "Plumes" ou "Bazars", accroches polysémiques permettant de développer toute une série d'histoires liées aux collections et ouvrant sur une théorie implicite. La collection mène donc à un réservoir d'images à la fois associatives, poétiques et théoriques.

À quoi servent les collections d'ethnographie au XXIe siècle dans un projet de musée?

L'exposition développée dans la Villa offre une réponse à cette question, liée à une manière d'appréhender les collections et la part du monde qu'elles racontent. Nous avons en effet énoncé clairement le principe de redécouverte : nous nous sommes rendus dans nos dépôts comme s'il s'agissait de nouveaux terrains afin d'y rencontrer des objets susceptibles de nous dire des choses sur le monde que nous ne leur avions jamais fait dire ou que nous n'avions jamais perçues auparavant, à la fois parce que nous les regardions autrement et parce que notre appareil critique avait changé. Le décalage concerné provient en grande partie du dialogue instauré avec les objets acquis récemment. Cette interrogation forte sur notre rapport à la culture matérielle s'est enrichie de la certitude que nous ne pouvons plus faire abstraction de ses aspects immatériels. 

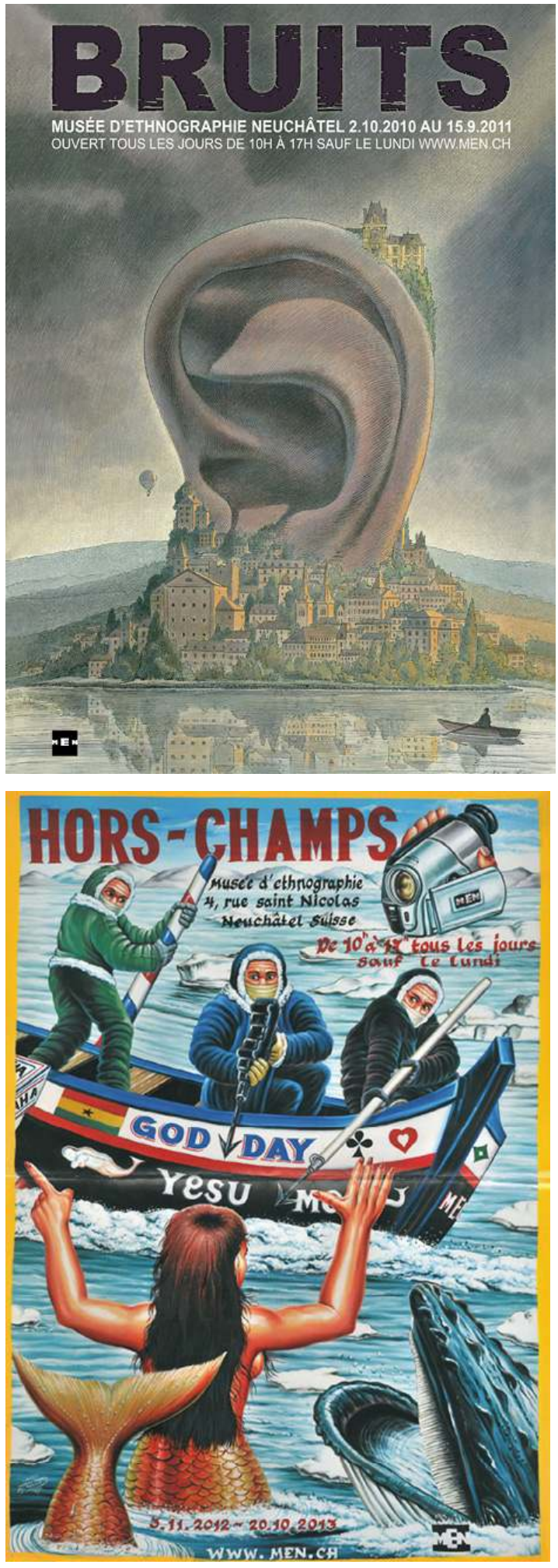

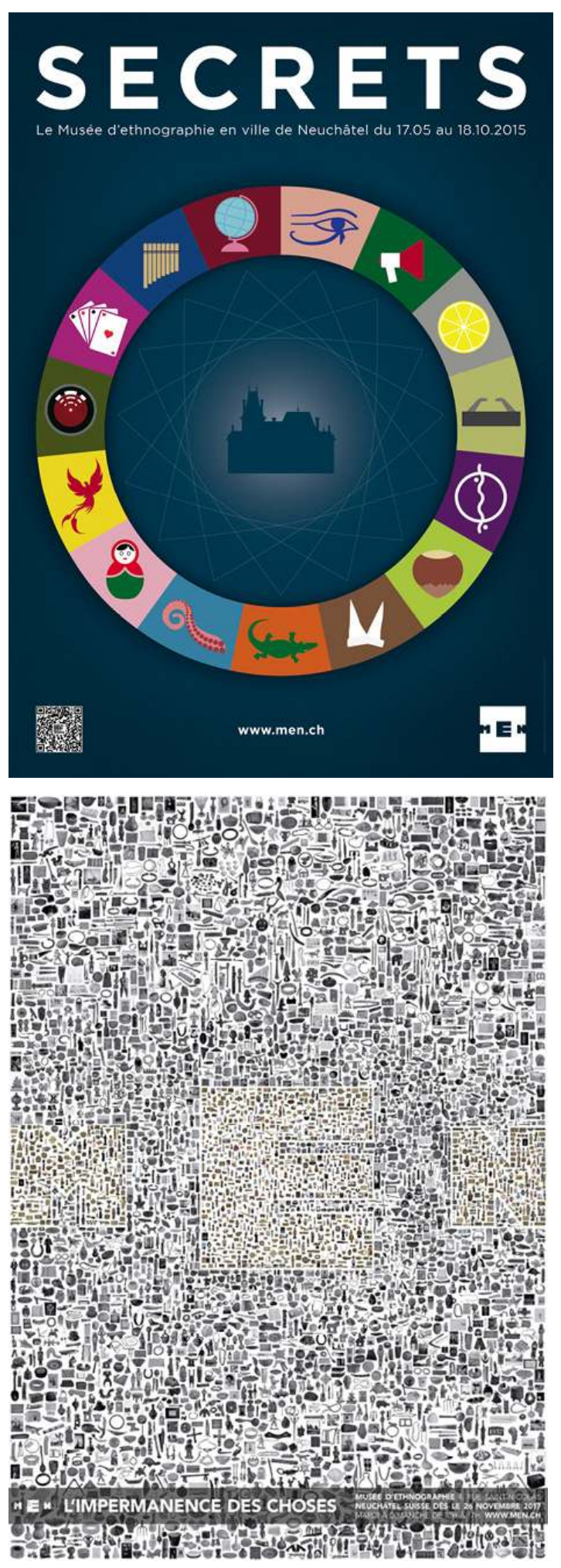

Votre approche d'une problématique très actuelle, celle des restitutions, est là encore très pertinente. Vous proposez une troisième voie.

38 Nous pensons en effet que nous ne sommes pas propriétaires des collections dont nous avons la responsabilité, mais que nous en sommes les gestionnaires transitoires. C'est la raison pour laquelle nous sommes intéressés par toute personne qui viendrait nous dire: " voilà, je sais que vous avez tel ou tel objet chez vous, il faudrait entrer en négociation pour savoir 
s'il ne pourrait pas être montré ailleurs, voire même rendu à une autre collectivité légitime". Vous avez compris la métaphore des coffres afghans dans le secteur "Ambassades" ? Ces coffres sont présentés dans une zone grillagée qui figure un abri de requérants d'asiles car ils ne sont pas destinés à rester à long terme au Musée d'ethnographie. disant que l'on ne comprenait rien chez nous ou que nous étions trop intellos. Je prétendais du reste le contraire, en disant que l'on mettait beaucoup d'éléments à disposition de notre public pour construire une visite, mais qu'en revanche on ne pouvait pas se mettre à la place du visiteur. C'est quand même à lui de faire l'effort de mise en relation, de faire le lien entre les divers éléments du discours. cela ne sert à rien d'expliquer de manière littérale et exhaustive le réel dans lequel nous sommes immergés. Il faut donc laisser au visiteur une part d'intuition, de sensibilité et de créativité dans son travail de reconstruction du sens. C'est sur ce plan que je reste fondamentalement élitaire : j'attends du visiteur qu'il ait le courage de se livrer à un exercice d'interprétation créative.

J'avais comme position de principe qu'une exposition doit se défendre toute seule et que c'est au visiteur de la découvrir grâce aux indices que nous lui donnons. Je me suis cependant rendu compte que la visite commentée proposait une autre manière d'appréhender notre travail, offrait une plateforme de communication et d'échanges susceptible de nous amener sur d'autres terrains. Je l'ai dès lors acceptée comme une expérience à part pour des personnes qui la demandaient. C'est donc là que j'ai le plus ouvert les 
vannes, en mettant notamment en place des brunchs mensuels associés à des visites guidées gratuites effectuées par les conservateurs.

Quels sont les retours, les réactions des publics, les perceptions suscitées depuis la réouverture du MEN?

Trois moments d'accueil et de restitution du MEN à la population locale ont été organisés, le premier avec les politiques et les sponsors, le deuxième avec les collègues d'ici et d'ailleurs et le troisième lors de deux journées complètes d'accueil de tous nos autres invités. L'exercice s'est avéré particulier car nous n'inaugurions pas seulement une nouvelle exposition de référence, mais proposions la redécouverte complète de la Villa. Le premier challenge était en effet de récréer un outil de travail solide. Ce challenge apparaît derrière l'exposition et émerge à travers la force de la proposition muséographique. Cette Villa est en effet devenue un vrai musée. Avant, c'était un ensemble de salles proposant des installations désuètes et coupées de l'extérieur, sans régulation du climat, ainsi que des bureaux répartis à tous les étages. Maintenant, c'est un véritable espace d'exposition qui permet d'installer des projets à géométrie variable, avec plafond et plancher techniques, possibilité d'accrochage de panneaux et tubes et régulation du climat. Les bureaux ont été regroupés dans le grenier illuminé par un patio à ciel ouvert. Ce nouvel agencement fait donc partie de la réception enthousiaste que nous avons reçue, j'en suis certain. J'ai senti des visiteurs soulagés, apaisés, bienveillants. Ce bon départ s'est confirmé par la suite, me faisant du reste dire à titre de boutade qu'un tel unanimisme avait quelque chose d'effrayant.

L'impermanence des choses : le secteur "Poids" avec le tableau des poids ashanti à gauche et la cascade d'étiquettes à droite

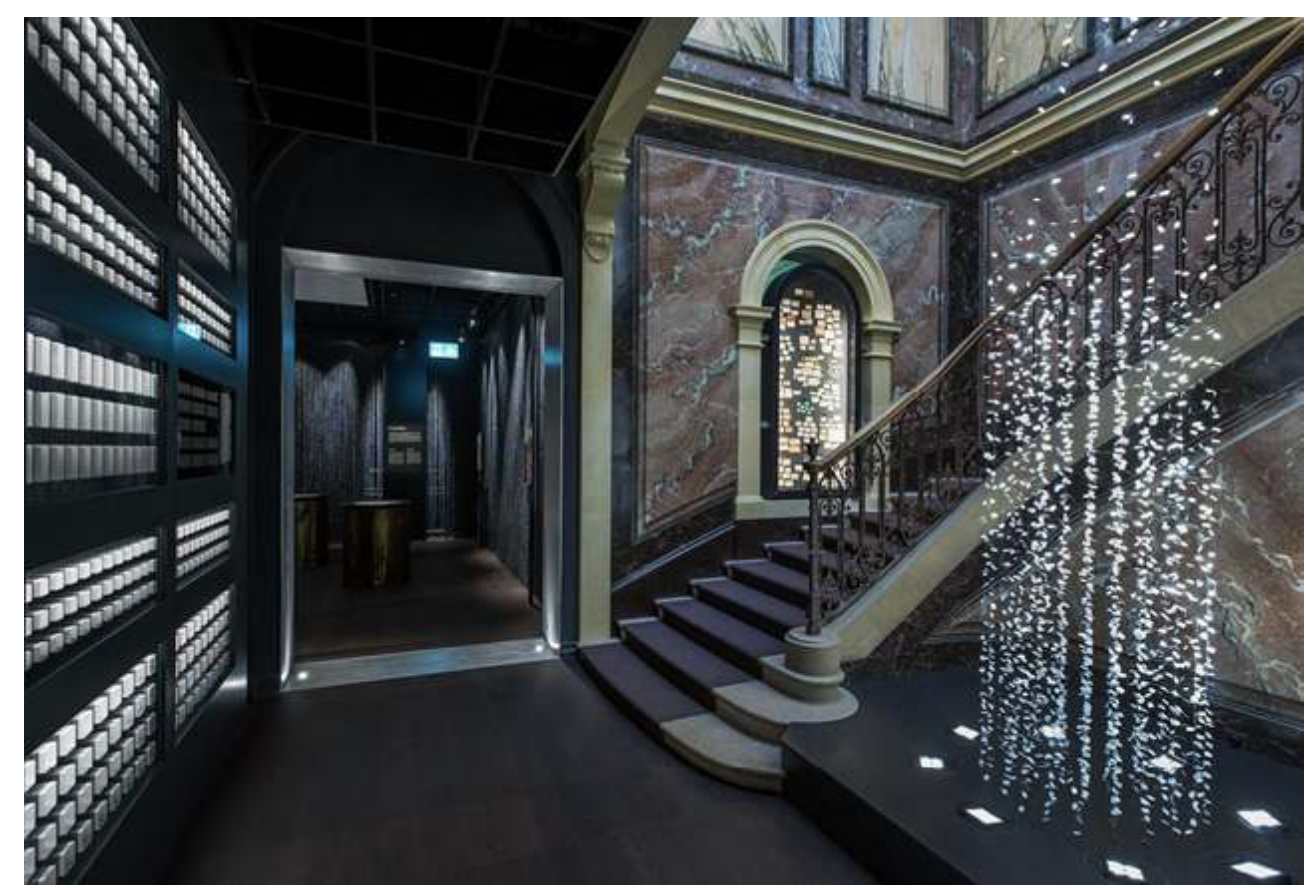

(c) MEN/Alain Germond 
L'impermanence des choses : la salle "Ambassades" avec les poupées soviétiques, les objets roumains et bulgares et les coffres afghans

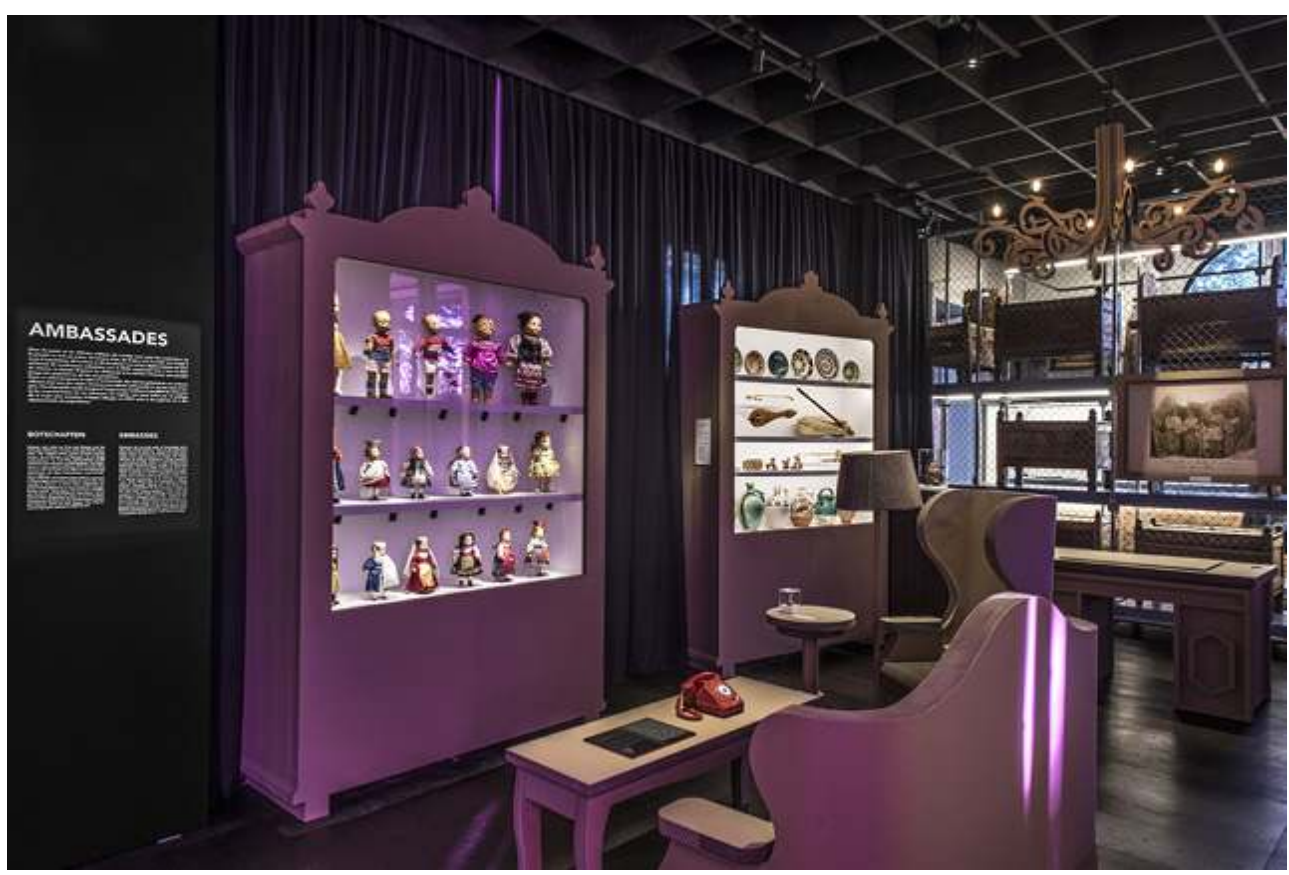

(C) MEN/Alain Germond

L'impermanence des choses : la salle "Artistes" avec au centre Ganesh de Zaven Paré, à droite et à gauche des œuvres de Bochimans du Botswana et au fond des enseignes de petits métiers d'Afrique de l'Ouest

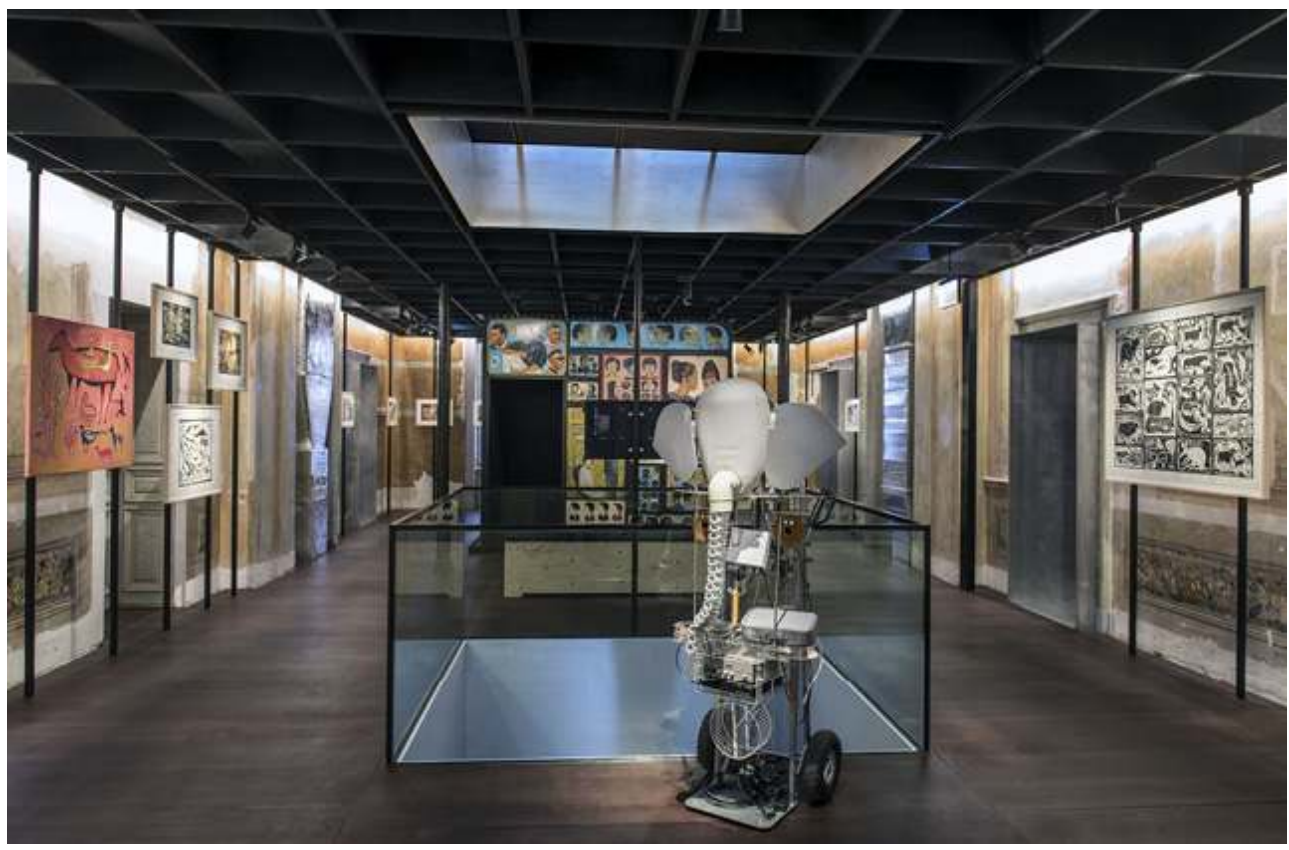

(c) MEN/Alain Germond

Vos affiches aussi ont contribué à la reconnaissance du musée et de ses expositions, ce sont toujours des images fortes. Ce processus fait partie de l'exposition et de la marque de fabrique du MEN. 
51 Il est fondamental. De mon côté, tant que nous n'avons pas une bonne affiche, je suis super inquiet. Celle de L'impermanence des choses fait référence à d'anciennes propositions du scénographe Jean-Pierre Zaug, qui a conçu plusieurs affiches associant des dizaines d'objets. Mais cette fois il y en a 4700 et le jeu conceptuel proposé renvoie aux dix ans de travail qui ont précédé la réouverture. Le logo au centre de l'affiche contient les objets intégrés dans l'exposition, apparaissant en couleur et donc presque en relief. Le fond est constitué d'objets en noir et blanc représentant un dixième des collections restantes, choisies de manière aléatoire et sans doublons dans les cinq continents puis disposés un à un par notre graphiste. vécue. Il a d'abord fallu trouver un titre, ce qui n'est pas venu rapidement. Il a ensuite fallu de nombreux essais manqués et une errance de plusieurs mois pour arriver à nos fins. Nous sommes alors revenus à l'idée de mosaïque, apparue très tôt dans le projet d'exposition, et à ce qui pourrait constituer cette mosaïque, à savoir les collections. Pour ne pas proposer une simple accumulation d'objets, il a ensuite fallu déterminer un élément central, la forme, et un élément offrant un fond à cette forme. La réponse est venue lors d'une séance que j'ai eue avec le graphiste chargé de l'affiche : l'objet central serait le logo et le fond serait constitué des collections. Restait à attribuer couleur et objets liés à l'exposition au logo et grisé et objets stockés dans nos dépôts au fond. Le reste ne fut que du tri, du travail et de l'ajustement. Ce processus me fait dire qu'une affiche reste essentiellement un objet pensé. Mais une fois l'affiche finalisée, vous disposez d'une vraie carte de visite et pouvez envisager de diffuser votre message sereinement.

Ce n'est absolument pas courant de donner un titre, L'impermanence des choses, à une exposition permanente.

Parce que ce n'en est pas une ! Il n'y a donc aucune contradiction. Le syntagme L'impermanence des choses... a été énoncé par un collègue lors d'une séance de travail. Je l'ai attrapé au vol et j'ai lancé: "ça c'est un titre pour notre exposition".

On n'a pas parlé de la mémoire des expositions réalisées au MEN. Les catalogues du MEN sortent plusieurs semaines, voire plusieurs mois après l'ouverture. Alors qu'en France, les catalogues sont faits pour le soir de l'inauguration.

C'est le mode que nous avons choisi pour nos catalogues. La raison pour laquelle on est toujours décalés, c'est que nous tenons à intégrer la mise en espace finale de l'exposition dans le catalogue. Et cela ne peut se faire avant l'ouverture car c'est le chaos jusqu'à la dernière semaine. En général, on a travaillé tellement vite pour terminer l'exposition que l'on n'a pas forcément fixé tous les contenus. Et comme on attend le corpus de photographies finales pour ajuster l'ensemble des textes, nous ne pouvons éditer le catalogue qu'après l'ouverture. Il faut ensuite une bonne semaine au photographe pour faire le tour des secteurs et nous sommes rarement disponibles tout de suite pour l'édition. Le catalogue sort donc généralement plusieurs mois après l'inauguration. Mais comme nos projets sont exposés assez longtemps... est réalisé, le catalogue parle aussi de la démarche de conception d'exposition.

On ne l'a jamais autant fait que dans le catalogue de Figures de l'artifice. On avait besoin d'expliciter la démarche que l'on avait suivie. Après, au fil des années, on s'est un petit 
peu éloignés de la matrice de base, même si nous avons presque toujours inclus des éléments liés au chantier. C'est quelque chose qui est resté jusqu'ici et cela sera aussi le cas dans le livre que nous sommes en train d'imprimer sur L'impermanence des choses. Des images de l'exposition finale ont été intégrées, de même que des documents liés à la conception et à la construction, rarement diffusés par nos collègues, même sur les sites internet de leurs établissements.

\section{RÉSUMÉS}

En décembre dernier, les espaces permanents du musée d'Ethnographie de Neuchâtel (MEN) étaient inaugurés dans la Villa de Pury, magnifiée par l'architecte Guido Pietrini. Conduite sous la direction de Marc-Olivier Gonseth, directeur de l'Institution, la rénovation surprend par son audace. Si le MEN est clairement à part dans le paysage des musées d'ethnographie européens, pour l'orientation de ses expositions temporaires, la transformation de la Villa et son exposition de référence sont bien de la même veine.

Après dix ans de direction, Marc-Olivier Gonseth a passé le relais fin avril à deux membres de son équipe, Yann Laville et Grégoire Mayor, qui assureront désormais la direction conjointe de cet établissement. Ainsi ce musée qui a marqué l'histoire de la muséographie continue et continuera, nous en prenons le pari, d'innover.

Nous publions ici quelques morceaux choisis. Retrouvez l'entretien dans son intégralité sur le site www.ocim.fr, rubrique La Lettre de l'Ocim. l'art. 